\title{
Three-dimensional multi-fluid model of a coronal streamer belt with a tilted magnetic dipole
}

\author{
L. Ofman ${ }^{1,2,3}$, E. Provornikova ${ }^{1,2}$, L. Abbo ${ }^{4}$, and S. Giordano ${ }^{4}$ \\ ${ }^{1}$ Department of Physics, Catholic University of America, Washington, DC 20064, USA \\ ${ }^{2}$ NASA Goddard Space Flight Center, Code 671, Greenbelt, MD 20771, USA \\ ${ }^{3}$ Visiting Associate Professor, Department of Geosciences, Tel Aviv University, Tel Aviv, Israel \\ ${ }^{4}$ INAF-Astrophysical Observatory of Torino, via Osservatorio 20, 10025 Pino Torinese, Italy \\ Correspondence to: L. Ofman (leon.ofman@nasa.gov)
}

Received: 1 September 2014 - Revised: 27 November 2014 - Accepted: 1 December 2014 - Published: 12 January 2015

\begin{abstract}
Observations of streamers in extreme ultraviolet (EUV) emission with SOHO/UVCS show dramatic differences in line profiles and latitudinal variations in heavy ion emission compared to hydrogen Ly- $\alpha$ emission. In order to use ion emission observations of streamers as the diagnostics of the slow solar wind properties, an adequate model of a streamer including heavy ions is required. We extended a previous 2.5-D multi-species magnetohydrodynamics (MHD) model of a coronal streamer to 3-D spherical geometry, and in the first approach we consider a tilted dipole configuration of the solar magnetic field. The aim of the present study is to test the 3-D results by comparing to previous $2.5-\mathrm{D}$ model result for a 3-D case with moderate departure from azimuthal symmetry. The model includes $\mathrm{O}^{5+}$ ions with preferential empirical heating and allows for calculation of their density, velocity and temperature in coronal streamers. We present the first results of our 3-D multi-fluid model showing the parameters of protons, electrons and heavy ions $\left(\mathrm{O}^{5+}\right)$ at the steady-state solar corona with a tilted steamer belt. We find that the 3-D results are in qualitative agreement with our previous 2.5-D model, and show longitudinal variation in the variables in accordance with the tilted streamer belt structure. Properties of heavy coronal ions obtained from the 3-D model together with EUV spectroscopic observations of streamers will help understanding the 3-D structures of streamers reducing line-of-sight integration ambiguities and identifying the sources of the slow solar wind in the lower corona. This leads to improved understanding of the physics of the slow solar wind.
\end{abstract}

Keywords. Solar physics astrophysics and astronomy (corona and transition region)

\section{Introduction}

White-light images of the solar corona from ground observations during the total solar eclipses and space-based coronagraphs show that the dominant magnetic structures of the minimum corona are equatorial streamers. These structures are usually seen as bright helmet-shaped features and characterized by closed magnetic field lines in their core and open field lines at outer edges (Gosling et al., 1981). The magnetic structure determines plasma parameters in the streamer, particularly high plasma density and electron temperature with a maximum at the core of the streamer. It has been well known for decades that, during the solar minimum, the magnetic field of the Sun is qualitatively described as a tilted dipole and a long-lived (several days or longer) quiescent streamer belt forms at low latitudes, as seen in eclipse images and white-light coronagraph observations. The streamer belt has been identified as the source of the slow solar wind by tracing the outflow of emerging small-scale structures ("blobs") in white light in the range 2-6 $R_{\mathrm{S}}$ (e.g., Sheeley et al., 1997). The streamer belt structures is also evident in UV emission of ions (e.g., Giordano and Mancuso, 2008).

The Ultraviolet Coronagraph Spectrometer (UVCS) onboard the Solar and Heliospheric Observatory (SOHO) spacecraft measures extreme ultraviolet (EUV) emissions produced by minor heavy ions of coronal plasma such as $\mathrm{O}^{5+}, \mathrm{Mg}^{9+}$ and $\mathrm{Si}^{10+}$. During the solar minimum, the structure of the solar corona observed in heavy ion emission differs considerably from the structure seen in white-light images and H I Ly- $\alpha$ emission (Kohl et al., 1997; Raymond et al., 1997; Noci et al., 1997). At the core of the streamer, 
the intensity of the H I Ly- $\alpha$ emission has a maximum; however emission from $\mathrm{O}^{5+}$ and $\mathrm{Mg}^{9+}$ ions is depleted and has a maximum at the edge of the streamer. This dramatic difference was clearly observed by UVCS during the solar minimum on 26 September 1997 (Ofman et al., 2011, 2013). Open magnetic field regions adjacent to streamer boundaries were found to be sources of the slow solar wind through abundance variations (e.g., Ofman, 2000; Antonucci et al., 2006; Ko et al., 2008). Observed variations in heavy ion emission are related to the variations in the ion abundances in the slow solar wind across the streamer (Chen and Li, 2004; Morgan et al., 2008; Ofman et al., 2011).

In order to use EUV coronal observations for diagnostics of the slow solar wind outflowing from streamers, a model of a streamer accounting for multi-component composition (protons, electrons, minor heavy ion species) of plasma is required. Single-fluid models are unable to describe the properties of heavy ions in the corona self-consistently. Multicomponent models of a coronal streamer that include plasma protons, electrons and heavy ions $\left(\mathrm{O}^{5+}\right.$ and $\left.\mathrm{Mg}^{9+}\right)$ were previously developed in Ofman et al. $(2011,2013)$. The plasma components are modeled as magnetized fluids coupled by collisions and electromagnetic interactions. Initial dipole configuration of the solar magnetic field and azimuthal (two spatial dimensions and three components of velocity and magnetic field) symmetry were assumed in these models. Ofman et al. $(2011,2013)$ showed a formation of an equatorial streamer belt; described latitudinal variations in parameters of protons, electrons and heavy ions; and obtained a good qualitative agreement between the calculated EUV emissions from the streamer using model output that compares well with UVCS observations in O VI and Mg X emissions.

The aim of our present study is to extend previous $2.5-\mathrm{D}$ multi-fluid models of streamers to 3-D geometry. Only a few 2.5-D multi-fluid model studies of multi-ion coronal streamers have previously been published (e.g., Ofman, 2000, 2004; Li et al., 2006; Ofman and Kramar, 2010; Ofman et al., 2011, 2013). The 3-D multi-fluid models allow for properties of coronal heavy ions to be studied in more realistic configurations of the coronal magnetic field, including the solar maximum conditions compared to the simplified dipole field considered in Ofman et al. (2011, 2013), and properly consider line-of-sight emission integration effects on the observed images. In the first approach we assume a tilted dipole configuration, which serves as a good approximation for the structure of solar magnetic field during the solar minimum (Kramar et al., 2009; Zhao and Hoeksema, 2010).

To fully explain the EUV data, it is necessary to apply sophisticated models of the corona that use observational data as input, such as a realistic coronal magnetic field configuration obtained from a potential-field source-surface (PFSS) model (Altschuler and Newkirk, 1969), which extrapolates the observed photospheric field (magnetograms). More realistic solar boundary conditions and initial state in the 3-D multi-fluid model are currently under development, and the results will be reported in future papers.

\section{Description of the model}

As in previous works, the model of a streamer includes heavy ion population treated as an additional fluid to plasma electron and proton fluids. In this paper we report the results of the model with $\mathrm{O}^{5+}$ ions. In the model, plasma is described using a three-fluid approximation - treating electrons, protons, and $\mathrm{O}^{5+}$ ions as coupled fluids through collisional and electromagnetic interactions - that considerably extends the single-fluid magnetohydrodynamics (MHD) description. We assume that plasma is quasi-neutral and neglect electron inertia, using $m_{\mathrm{e}} \ll m_{\mathrm{p}}$ to solve the electron momentum equation for the electric field. Density of electrons is obtained from charge neutrality condition. Viscosity and explicit thermal conduction terms are ignored in this model. The following equations in normalized form are solved:

$$
\begin{aligned}
\frac{\partial n_{k}}{\partial t}=-\nabla \cdot\left(n_{k} \boldsymbol{V}_{k}\right), \\
n_{k}\left[\frac{\partial \boldsymbol{V}_{k}}{\partial t}+\left(\boldsymbol{V}_{k} \cdot \nabla\right) \boldsymbol{V}_{k}\right]=-E u_{k} \nabla p_{k}-E u_{\mathrm{e}} \frac{Z_{k} n_{k}}{A_{k} n_{\mathrm{e}}} \nabla p_{\mathrm{e}} \\
\quad-\frac{n_{k}}{F r r^{2}} \boldsymbol{e}_{\mathrm{r}}+\Omega_{k} n_{k}\left(\boldsymbol{V}_{k}-\boldsymbol{V}_{\mathrm{e}}\right) \times \boldsymbol{B}+\boldsymbol{F}_{v}+n_{k} \boldsymbol{F}_{k, \mathrm{coul}},
\end{aligned}
$$

$$
\frac{\partial \boldsymbol{B}}{\partial t}=\nabla \times\left(\boldsymbol{V}_{\mathrm{e}} \times \boldsymbol{B}\right)+\frac{1}{S} \nabla^{2} \boldsymbol{B},
$$

$$
\boldsymbol{V}_{\mathrm{e}}=\frac{1}{n_{\mathrm{e}}}\left(n_{\mathrm{p}} \boldsymbol{V}_{\mathrm{p}}+Z_{\mathrm{i}} n_{\mathrm{i}} \boldsymbol{V}_{\mathrm{i}}-b \nabla \times \boldsymbol{B}\right),
$$

$$
\begin{gathered}
\frac{\partial T_{k}}{\partial t}=-\left(\gamma_{k}-1\right) T_{k} \nabla \cdot V_{k}-\boldsymbol{V}_{k} \cdot \nabla T_{k} \\
+C_{k j l}+\left(\gamma_{k}-1\right)\left(S_{k}-\delta_{k, \mathrm{e}} S_{\mathrm{r}, \mathrm{e}}\right),
\end{gathered}
$$

where the index $k=\mathrm{p}$, $\mathrm{i}$ for protons and heavy ions (in the energy Eq. $5 k=$ e, p, i including electrons), $n_{k}$ is the number density, $\boldsymbol{V}_{k}$ is the velocity vector, $T_{k}$ is the temperature for each species; $S_{k}$ is the empirical heating term (used only for heavy ions), $S_{\mathrm{r}, \mathrm{e}}$ is the electron radiative loss term (we use the same function as in Ofman et al., 2011), $\delta_{k, \mathrm{e}}$ is the Kronecker delta, $C_{k j l}$ is the energy coupling term between the species (for detailed expression see Ofman, 2004), and $\gamma_{k}$ is the polytropic index of each species. We assume polytropic index $\gamma_{k}=1.05$, neglecting explicit heating and heat conduction terms for electrons and protons for simplicity. This choice of $\gamma$ accounts for the (unknown) bulk heating of the corona and yields nearly isothermal flow of electrons and protons. This approach and the value of $\gamma$ are often used 
in global modeling of the solar wind (e.g., Riley et al., 2006). $A_{k}$ is the mass number and $\Omega_{k}=\frac{Z_{k} e B_{0}}{A_{k} m_{\mathrm{p}} c} \tau_{\mathrm{A}}$ is the normalized gyrofrequency, where $m_{\mathrm{p}}$ is the proton mass and $\tau_{\mathrm{A}}$ is the Alfvén time $\tau_{\mathrm{A}}=R_{\mathrm{S}} / V_{\mathrm{A}}$, where $V_{\mathrm{A}}=B_{0} / \sqrt{4 \pi m_{\mathrm{p}} n_{\mathrm{e} 0}}$ is the Alfvén speed (defined here with the proton mass), $B_{0}$ is the normalization magnetic field strength (in the present study $\left.B_{0}=7 G\right), m_{\mathrm{p}}$ is the proton mass, and $n_{\mathrm{e} 0}$ is the normalization value of the electron number density (we use $n_{\mathrm{e} 0}=5 \times 10^{8} \mathrm{~cm}^{-3}$, and therefore $V_{\mathrm{A}}=683 \mathrm{~km} \mathrm{~s}^{-1}$ ).

Normalization used in the above equations is the same as described in Ofman et al. (2011). The dimensionless parameters in the system are the Lundquist number $S$ (in the present study $10^{4}$ ); electron and proton Euler number $E u_{\mathrm{e}, \mathrm{p}}=$ $\left(k_{\mathrm{b}} T_{0, \mathrm{e}, \mathrm{p}} / m_{\mathrm{p}}\right) V_{\mathrm{A}}^{-2}$; ion Euler number $E u_{\mathrm{i}}=\left(k_{\mathrm{b}} T_{0, \mathrm{i}} / m_{\mathrm{i}}\right) V_{\mathrm{A}}^{-2}$; and Froude number $F r=V_{\mathrm{A}}^{2} R_{\mathrm{S}} / G M_{\mathrm{S}}$, where $G$ is the universal gravitational constant and $M_{\mathrm{S}}$ is the solar mass, $R_{\mathrm{S}}$ is the solar radius, $b=c B_{0} /\left(4 \pi e n_{\mathrm{e} 0} R_{\mathrm{S}} V_{\mathrm{A}}\right)$ the normalization constant, and $k_{\mathrm{b}}$ is the Boltzmann constant. The Coulomb friction terms $\boldsymbol{F}_{k \text {,coul }}$ are given by Braginskii (1965) (see also Li et al., 1997; Ofman, 2004, for the detailed expressions of these terms).

The heating term for $\mathrm{O}^{5+}$ ions is the function $S_{\mathrm{i}}=S_{\mathrm{i} 0}(r-$ 1) $e^{-(r-1) / \lambda_{\mathrm{i} 0}}$, where the constants $S_{\mathrm{i} 0}$ and $\lambda_{\mathrm{i} 0}$ determine the magnitude and the decrease of the heating rate with distance $r$. These parameters are chosen to match the radial dependence of $\mathrm{O}^{5+}$ ion temperature retrieved from EUV observations. We adopt the values used in our previous 2.5-D study: $S_{\mathrm{i} 0}=29.0$ and $\lambda_{\mathrm{i} 0}=0.5$. The temperature anisotropy of the $\mathrm{O}^{5+}$ ion temperature may become significant in streamers, with observational evidence exhibiting considerable uncertainties (e.g., Frazin et al., 2003). As in our previous 2.5-D multi-fluid studies, for simplicity we neglect the effects of possible temperature anisotropy; we plan to address this in future studies.

We solve the equations numerically in the 3-D spherical domain, where spatial coordinates ranges are $R_{\mathrm{S}} \leq r \leq 8 R_{\mathrm{S}}$, $0<\theta<\pi$ and $0 \leq \phi \leq 2 \pi$. We initialize the computations with the tilted dipole coronal magnetic field, where the angle between the magnetic axis and solar rotation axis is $10^{\circ}$ (similar to the formalism of Finlay et al., 2010, for the Earth's tilted dipole). The low variability in $\phi$ of the tilted dipole allows for the use of a lower resolution compared to a more complex magnetic field, and is favorable for testing the model. Initial proton velocity $V_{\mathrm{p}, \mathrm{r}}$ and density $n_{\mathrm{p}}$ in the domain are obtained from an analytic Parker isothermal solar wind solution (Parker, 1963) with $T_{0, \mathrm{p}}=1.6 \mathrm{MK}$. The initial $\mathrm{O}^{5+}$ density is taken as $8 \times 10^{4} n_{\mathrm{p}}$ at $r=1 R_{\mathrm{S}}$ which corresponds to total oxygen relative abundance. An initially uniform $\mathrm{O}^{5+}$ temperature $T_{\mathrm{O}^{5+}}=32 \mathrm{MK}$ and a hydrostatic distribution of oxygen with height was used to initialize the calculations. Ion fractionation ratio can be applied to the solutions to obtain the $\mathrm{O}^{5+}$ relative abundance and calculate the emissivity (see, Ofman et al., 2011). Note that the large initial heavy ion fluid temperature facilitates the initiation of

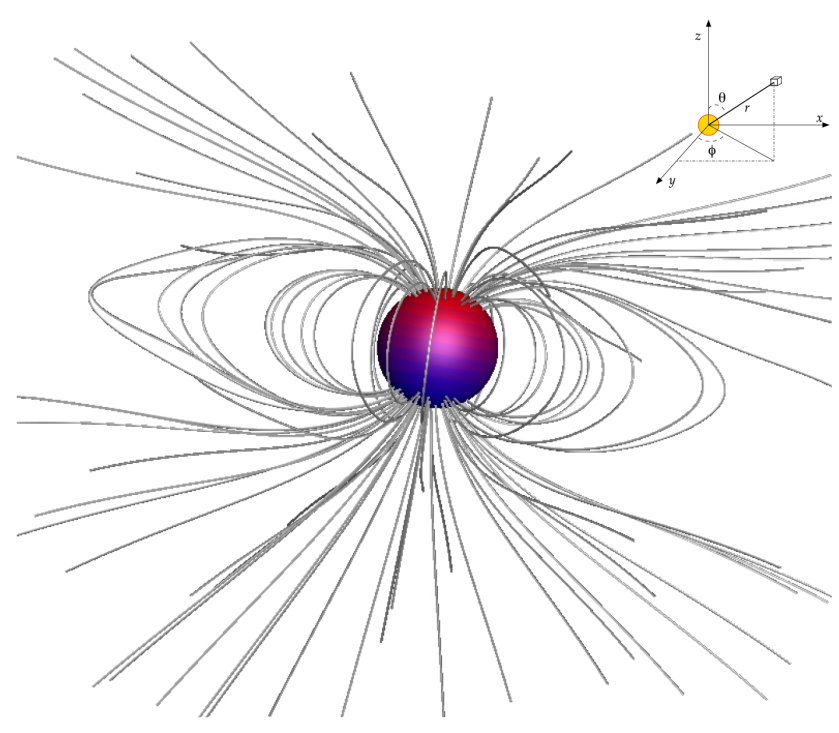

Figure 1. Three-dimensional structure of the coronal magnetic field in the steady-state solution showing formation of a streamer belt from the initial tilted dipole magnetic field configuration. The coordinate system is shown in the top right corner.

the calculations by extending the heavy ion initial gravitational scale height, but the oxygen ion temperature decreases rapidly as the streamer is formed through cooling by coupled electron and proton fluids.

The following boundary conditions are applied. At the inner boundary $r=1 R_{\mathrm{S}}$, the magnetic field components are fixed and values of density, temperature and velocity components are extrapolated from interior cells with zero gradients (extrapolated boundary conditions approximating incoming characteristics based on the method described in Steinolfson and Nakagawa, 1976). At the outer boundary $r=8 R_{\mathrm{S}}$, open boundary conditions are set for all variables. The results shown below were obtained with a resolution of $160 \times 128 \times 512$ grid cells in the $\theta, \phi$ and $r$ directions, respectively. We have performed test runs and found no significant difference in solutions with higher resolutions of $320 \times 128 \times 512$ and $160 \times 256 \times 512$.

The equations are solved using a fourth-order RungeKutta method in time and fourth-order spatial differencing. A Chebyshev fourth-order smoothing term is applied for stability. The code is parallelized using the Message Passing Interface with an efficient solution on 256 processors.

\section{Results and discussion}

Figures 1-5 show results of the 3-D multi-fluid simulation of a streamer with electrons, protons and $\mathrm{O}^{5+}$ ions. The numerical solution is evolved until a (quasi-)steady state is reached, with the typical closed field and open field region structures separated by a current sheet in the streamer. At this stage temporal changes of the solution are very small compared to 


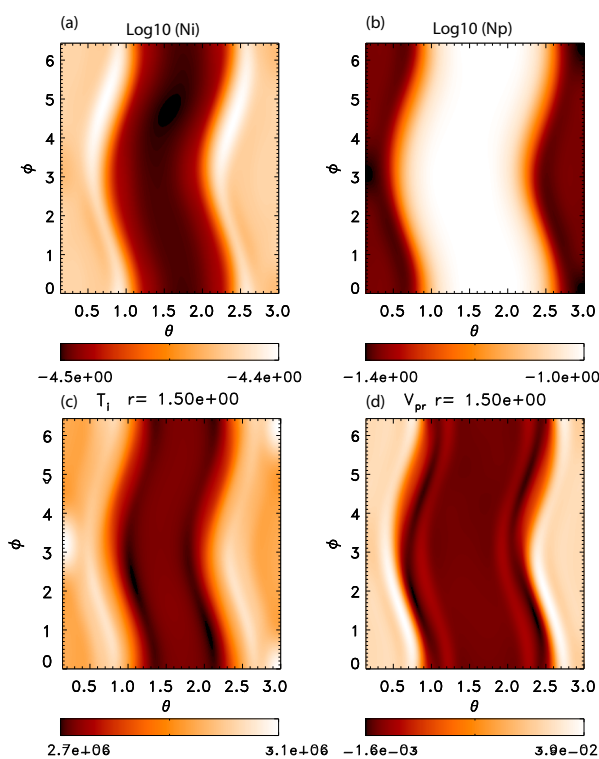

Figure 2. Maps of (a) $\mathrm{O}^{5+}$ ion density ( $\log _{10}$ scale), (b) proton density $\left(\log _{10}\right.$ scale of the normalized density by $\left.n_{\mathrm{e} 0}=5 \times 10^{8} \mathrm{~cm}^{-3}\right)$, (c) $\mathrm{O}^{5+}$ ion temperature $[\mathrm{K}]$, and (d) radial proton velocity on a Carrington map at $r=1.5 R_{\mathrm{S}}(\theta-\phi$ plane) obtained from a $3-\mathrm{D}$ three-fluid model for a tilted dipole solar magnetic field. The velocities are in units of $V_{\mathrm{A}}=683 \mathrm{~km} \mathrm{~s}^{-1}$.

variations at the beginning of the simulation. To find a steady state, we examined temporal evolution of the variables such as densities, velocities and magnetic field in the 2-D plane cuts of the 3-D solution, as well as at several points in the computational domain. Figure 1 presents a 3-D view of magnetic field lines at the steady-state solution. The formation of a tilted equatorial streamer belt can be seen.

Figure 2 shows logarithmic density maps of $\mathrm{O}^{5+}$ ions and protons (panels $\mathrm{a}$ and $\mathrm{b}$ ), and the corresponding ion temperature (panel c) and proton radial velocity (panel d) in the $\phi-\theta$ plane at $r=1.5 R_{\mathrm{S}}$. The corresponding latitudinal profiles of radial velocity and density are presented in Fig. $4 \mathrm{a}$ and $\mathrm{b}$. It can be seen that the proton density is enhanced at the core of the streamer, while the $\mathrm{O}^{5+}$ ion density is depleted at the core and has maxima at the boundary separating open and closed field regions. The proton density is larger inside the streamer by a factor of 2.5 compared to the density outside. The $\mathrm{O}^{5+}$ ion density in the core of the streamer is smaller by $30 \%$ than outside, while the $\mathrm{O}^{5+}$ ion temperature is lower inside the streamer. These results are consistent with previous 2.5-D models of the streamer and with UVCS observations of $\mathrm{O}^{5+}$ and $\mathrm{Mg}^{9+}$ ions emission from quiescent streamers during the solar minimum (Ofman et al., 2011). The minimum of $\mathrm{O}^{5+}$ density at the streamer core is the result of gravitational settling of ions in the closed field region. While the coupling between the outflowing protons and $\mathrm{O}^{5+}$ ions due to Coulomb friction in the open field region produces the increase in the ion abundance, the cooling of the ions from their initial temperature through thermal coupling with elec-

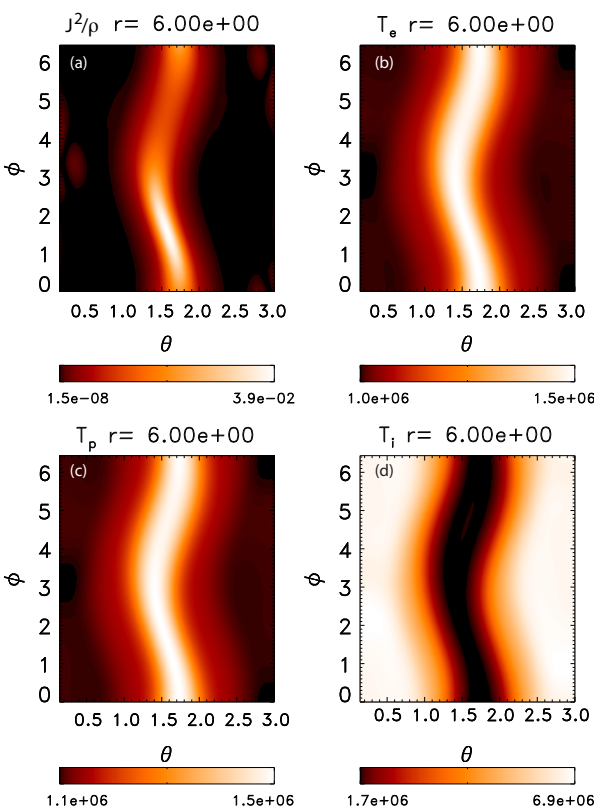

Figure 3. (a) The normalized current density $j^{2} / \rho$ obtained from the model at $6 R_{\mathrm{S}}$. (b), (c) and (d) show the temperature maps in kelvin in the $\theta-\phi$ plane at $r=6 R_{\mathrm{S}}$ for electrons, protons, and $\mathrm{O}^{5+}$ ions, respectively, that correspond to the quasi-steady state of the streamer belt at the end of the run.

trons and protons further enhances gravitational settling in the core of the streamer and reduces the ion relative abundance. Also, as expected in the streamer, the density of protons and $\mathrm{O}^{5+}$ ions decreases with height. Plots of radial speed of protons and heavy ions show that at the streamer core the speeds are nearly zero, while the outflow forms in the regions with open magnetic field lines, consistent with UVCS observations (e.g., Strachan et al., 2002; Antonucci et al., 2005; Abbo et al., 2010).

Figure 3 shows maps of temperature of electrons, protons and $\mathrm{O}^{5+}$ ions (panels b, c, d, respectively) at the distance $r=6 R_{\mathrm{S}}$. Latitudinal temperature profiles at $r=1.6 R_{\mathrm{S}}$ are shown in Fig. 4c. Figure 5 shows the streamer structure in several variables in the $r-\theta$ plane at $\phi=0.76 \mathrm{rad}$. The ion and proton density, the electron and ion temperatures, the proton radial outflow speed, and the normalized current density are shown. The streamer structure is evident and is similar to the 2.5-D results in Ofman et al. (2011), with the main difference evident in the tilt, i.e., the streamer symmetry axis is shifted to higher latitude at this longitude compared to the non-tilted case. This can contribute to the line-of-sight integration of the streamer when observed in EUV in the plane of the sky by showing angularly broader streamer structure by up to the tilt angle. Electron and proton temperatures are nearly equal and have a maximum value at the core of the streamer compared to its boundaries, which is consistent with observations of a coronal streamer at the solar minimum and previous model results (Ofman et al., 2011, 2013). 

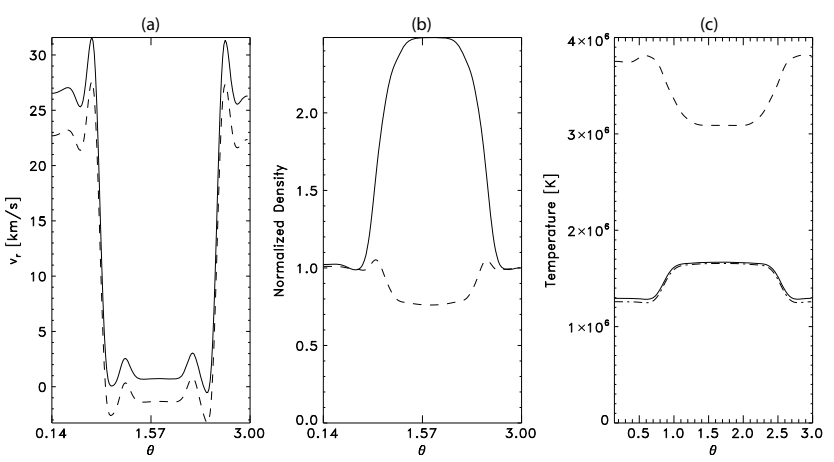

Figure 4. Latitudinal profiles of (a) radial speed, (b) normalized density and (c) temperatures for solar wind ions (dashed curves), protons (solid curves) and electrons (dash-dot curves) at $r=1.6 R_{\mathrm{S}}$ and $\phi=0.76 \mathrm{rad}$ at $t=56.1 \tau_{\mathrm{A}}$.

In the closed field regions of the streamer, the proton and electron temperatures are almost constant due to hydrostatic equilibrium of a streamer at the steady state. Meanwhile the $\mathrm{O}^{5+}$ temperature is depleted at the core due to cooling by electrons and protons with corresponding gravitational settling, and increases at the boundary of the streamer, where the heating effects are stronger compared to cooling. In the closed field region, hot $\mathrm{O}^{5+}$ ions and cooler protons and electrons exchange energy by collisions, which results in lower temperatures of $\mathrm{O}^{5+}$ ions compared to their temperature outside the closed field line region. The longitudinal variation of the velocity and density inhomogeneity at the boundary of the streamer due to the tilted magnetic configuration and corresponding variation in the magnetic pressure is evident. Variations in temperature of the species across the streamer reflect latitudinal variations in magnetic field and density. Obtained temperature variations in $\mathrm{O}^{5+}$ ions in the streamer are consistent with UVCS spectroscopic observations of broad ion emission lines and previous 2.5-D model results for $\mathrm{O}^{5+}$ and $\mathrm{Mg}^{9+}$ ions. Since we assumed nearly isothermal energy equations for protons and electrons with polytropic index $\gamma_{\mathrm{e}}=\gamma_{\mathrm{p}}=1.05$ and no empirical heating for these species, variations in electron and proton temperature with height are small. For $\mathrm{O}^{5+}$ ions, radially dependent empirical enhanced heating as well as cooling due to interaction with protons and electrons is included in the energy equation. These processes result in an increase in $\mathrm{O}^{5+}$ temperature with height to about $3 R_{\mathrm{S}}$ and decrease at larger distances.

The current density structure, $j^{2} / \rho$, is shown in Fig. 3a in the $\theta-\phi$ plane at $r=6 R_{\mathrm{S}}$, and its height dependence is evident in Fig. 5f, showing a cut in the $\theta-r$ plane at $\phi=0.76 \mathrm{rad}$. The division by the total density $\rho$ counters the height dependence and shows the current density per unit mass (in normalized units). Since the stalk of the streamer has formed at this height, the current is confined to a narrow band where the magnetic field radial component is chang-
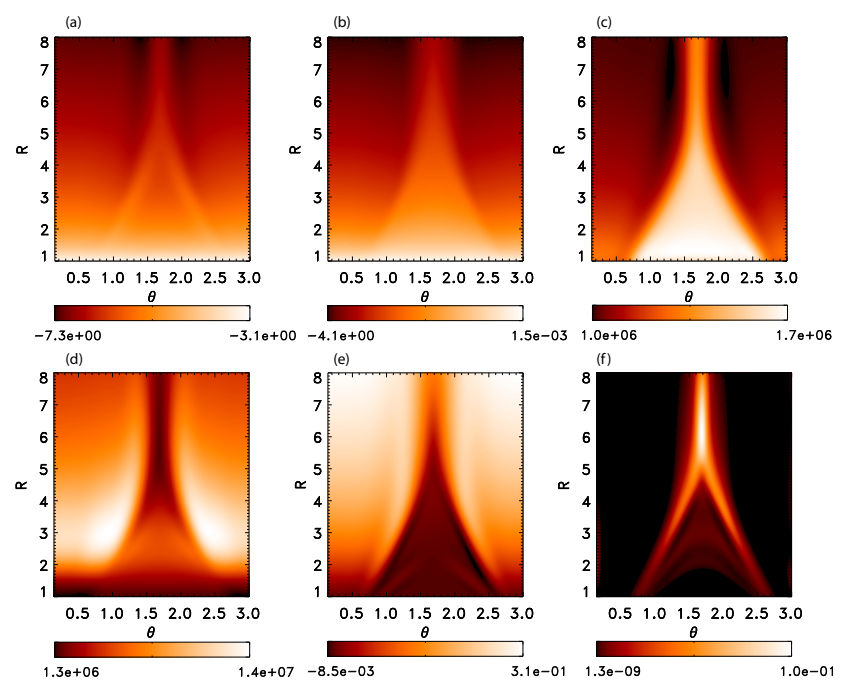

Figure 5. The variable in the $r-\theta$ plane at $\phi=0.76 \mathrm{rad}$ at $t=$ $54.08 \tau_{\mathrm{A}}$. (a) The $\mathrm{O}^{5+}$ ion density $\left(\log _{10}\right.$ scale), (b) the proton density $\left(\log _{10}\right.$ scale), (c) the electron temperature $T_{\mathrm{e}},(\mathbf{d})$ the $\mathrm{O}^{5+}$ temperature $T_{\mathrm{i}}$, (e) the proton radial velocity $V_{\mathrm{p}, \mathrm{r}}$ and (f) the normalized current density $j^{2} / \rho$.

ing sign. The width of the current layer is determined by the physical and numerical resistivity in the model. The longitudinal $(\phi)$ dependence of the tilted magnetic structure leads to second-order intensity variation in the current density due to longitudinal variation in the background magnetic pressure.

Overall the structure of the streamer obtained in the 3-D model is consistent with previously obtained results in 2.5D model (Ofman et al., 2011, 2013). The previous model showed a good agreement between the latitudinal profiles of proton and $\mathrm{O}^{5+}$ ion density and the temperature and values derived from UVCS observational data. Small velocities of protons and ions in the core of the streamer obtained in the 2.5-D model also agreed with UVCS velocity measurements (for detailed comparison see Ofman et al., 2011). Using results of the 2.5-D model, Ofman et al. (2011) computed maps of UV emission from streamers in lines H I Ly- $\alpha$ and O VI $1032 \AA$ and compared to intensity maps constructed from UVCS observations. A good agreement was found between the computed and observed structure of streamers seen in UV emission.

Our next step in a future study will be to compute the intensity maps using the results of a 3-D model presented above. By using the 3-D model, no geometrical assumptions are needed to integrate the emissivity along the line of sight, contrary to the intensity computation from the 2.5-D model. This will allow us to perform a quantitative comparison with observed UV and white-light coronal streamers from UVCS and LASCO coronagraphs aboard SOHO and from STEREO Cor1, as well as ground-based observations when a more realistic coronal field is incorporated into the model. 


\section{Conclusions}

During the solar minimum the structure of the solar magnetic field can be well approximated by the tilted dipole configuration. In this case a tilted single streamer belt forms with associated slow solar wind outflow. In the presented study we extend our previous time-dependent multi-component 2.5-D model of the solar streamer belt with azimuthal symmetry to full 3-D geometry, which ultimately allows for reduction of line-of-sight optically thin UV emission integration ambiguity effects in observed streamer images. The 3-D multifluid model allows for the properties of the slow solar wind as observed in spectral lines of minor heavy ions to be studied in a more realistic configuration of the coronal magnetic field. During the solar maxima conditions, when streamers may form at high latitudes, the line-of-sight ambiguity becomes even more important, affecting observations of optically thin ion emission. In this paper we report our first results of a three-fluid 3-D model of the streamer belt, and in the first step we considered the tilted dipole configuration of the coronal field. We assumed that, in addition to protons and electrons, coronal plasma contains hot (compared to protons and electrons) heavy $\mathrm{O}^{5+}$ ions consistent with past UVCS observations (e.g., Kohl et al., 1997; Raymond et al., 1997). The unknown ion heating process is modeled by an empirical heating term in the oxygen ion energy equation. Several simplifying assumptions are used in the model as noted in Sect. 2 above.

The structure of the streamer obtained in the 3-D model is consistent with results of the previous azimuthally symmetric 2.5-D model and with observations reported previously, with additional azimuthal variations in the variables. As expected, the model shows the formation of the tilted streamer belt with the following properties of plasma components:

1. Proton density is enhanced at the core of the streamer, while the $\mathrm{O}^{5+}$ ion density is depleted at the core and has a maximum value at the boundary of the streamer.

2. The radial speed of protons and $\mathrm{O}^{5+}$ ions is nearly zero at the core, while outflow forms at the edge of the streamer in the region with open magnetic field lines consistent with slow solar wind.

3. Electron and proton temperatures are equal at the core and the value is higher than outside the streamer.

4. $\mathrm{O}^{5+}$ ion temperature is higher than proton and electron temperatures in the streamer at the same height, while it is depleted at the core and increases at the boundary of the streamer.

We conclude that latitudinal variations in parameters of heavy ions are determined by energy and momentum exchange in the interaction of ions with protons and electrons. The longitudinal $(\phi)$ dependence of the streamer structure due to the tilt (warping) is evident. In particular, the density compression and rarefaction of the heavy ions and corresponding velocity variations at the boundaries of the streamer belt are evident at $1.5 R_{\mathrm{S}}$ (Fig. 2), and the second-order variation in the current density structure is most evident at $6 R_{\mathrm{S}}$ (Fig. 3a). The next step in our 3-D study will be to compute the UV intensity maps in H I Ly- $\alpha$ and O VI $1032 \AA$ lines using the plasma parameters of the streamer and to compare these to the maps from UVCS data, extending the 2.5D model of Ofman et al. $(2011,2013)$; furthermore, a more complex solar magnetic field and initial state consistent with solar maximum conditions will be included.

While significantly improved, the model still holds several limitations such the as use of polytropic energy equations with $\gamma=1.05$ (which allows for explicit terms for electron heat conduction, electron and proton heating to be neglected) and the use of a radially dependent empirical heating function for heavy ions. Our 3-D model allows for implementation of a realistic coronal magnetic field derived from a measured photospheric magnetic field, using the PFSS model as the initial state. The addition of further complexities to the model by including these effects will be the subject of future studies.

Acknowledgements. L. Ofman and E. Provornikova would like to acknowledge support provided through NSF grant AGS-1059838. L. Abbo would like to acknowledge support provided through contract I/023/09/0 between the National Institute for Astrophysics (INAF) and the Italian Space Agency (ASI). The computations were carried out at NASA Ames Advanced Supercomputing Division.

Topical Editor V. Fedun thanks the two anonymous referees for their help in evaluating this paper.

\section{References}

Abbo, L., Antonucci, E., Mikić, Z., Linker, J. A., Riley, P., and Lionello, R.: Characterization of the slow wind in the outer corona, Adv. Space Res., 46, 1400, doi:10.1016/j.asr.2010.08.008, 2010.

Antonucci, E., Abbo, L. and Telloni, D.: Slow wind and magnetic topology in the solar minimum corona in 1996-1997, Astron. Astrophys., 435, 699, doi:10.1051/0004-6361:20047126, 2005.

Antonucci, E., Abbo, L., and Telloni, D.: Oxygen Abundance and Energy Deposition in the Slow Coronal Wind, Astrophys. J., 643 , 1239, doi:10.1086/503186, 2006.

Altschuler, M. D. and Newkirk, G.: Magnetic fields and the structure of the solar corona, Sol. Phys., 9, 131, doi:10.1007/BF00145734, 1969.

Braginskii, S. I.: Transport Processes in a Plasma, in: Review of Plasma Physics, Consultants Bureau, New York, 205-311, 1965.

Chen, Y. and Li, X.: An Ion-Cyclotron Resonance-driven ThreeFluid Model of the Slow Wind near the Sun, Astrophys. J. Lett. 609, L41, doi:10.1086/422581, 2004.

Finlay, C. C., Maus, S., Beggan, C. D., Bondar, T. N., Chambodut, A., Chernova, T. A., Chulliat, A., Golovkov, V. P., Hamilton, B. Hamoudi, M., Holme, R., Hulot, G., Kuang, W., Langlais, B., 
Lesur, V., Lowes, F. J., Lühr, H., MacMillan, S., Mandea, M., McLean, S., Manoj, C., Menvielle, M., Michaelis, I., Olsen, N., Rauberg, J., Rother, M., Sabaka, T. J., Tangborn, A., TøffnerClausen, L., Thébault, E., Thomson, A. W. P., Wardinski, I., Wei, Z., and Zvereva, T. I.: International Geomagnetic Reference Field: the eleventh generation, Geophys. J. Int., 183, 1216, doi:10.1111/j.1365-246X.2010.04804.x, 2010.

Frazin, R. A., Cranmer, S. R., and Kohl, J. L.: Empirically Determined Anisotropic Velocity Distributions and Outflows of $\mathrm{O}^{5+}$ Ions in a Coronal Streamer at Solar Minimum, Astrophys. J., 597, 1145, doi:10.1086/378558, 2003.

Giordano, S. and Mancuso, S.: Coronal Rotation at Solar Minimum from UV Observations, Astrophys. J., 688, 656, doi:10.1086/591923, 2008.

Gosling, J. T., Asbridge, J. R., Bame, S. J., Feldman, W. C., Borrini, G., and Hansen, R. T.: oronal streamers in the solar wind at $1 \mathrm{AU}$, J. Geophys. Res., 86, 5438-5448, doi:10.1029/JA086iA07p05438, 1981.

Ko, Y.-K., Li, J., Riley, P., and Raymond, J. C.: Large-Scale Coronal Density and Abundance Structures and Their Association with Magnetic Field Structure, Astrophys. J., 683, 1168, doi:10.1086/589873, 2008.

Kohl, J. L., Noci, G., Antonucci, E., Tondello, G., Huber, M. C. E., Gardner, L. D., Nicolosi, P., Strachan, L., Fineschi, S., Raymond, J. C., Romoli, M., Spadaro, D., Panasyuk, A., Siegmund, O. H. W., Benna, C., Ciaravella, A., Cranmer, S. R., Giordano, S., Karovska, M., Martin, R., Michels, J., Modigliani, A., Naletto, G., Pernechele, C., Poletto, G., and Smith, P. L.: First Results from the Soho Ultraviolet Coronagraph Spectrometer, Sol. Phys., 175, 613-644, doi:10.1023/A:1004903206467, 1997.

Kramar, M., Jones, S., Davila, J., Inhester, B., and Mierla, M.: On the Tomographic Reconstruction of the 3D Electron Density for the Solar Corona from STEREO COR1 Data, Sol. Phys., 259, 109-121, doi:10.1007/s11207-009-9401-2, 2009.

Li, B., Li, X., and Labrosse, N.: A global 2.5-dimensional three fluid solar wind model with alpha particles, J. Geophys. Res., 111, 8106, doi:10.1029/2005JA011303, 2006.

Li, X., Esser, R., Habbal, S. R., and Hu, Y.-Q.: Influence of heavy ions on the high-speed solar wind, J. Geophys. Res., 102, 1741917432, doi:10.1029/97JA01448, 1997.

Morgan, H., Fineschi, S., Habbal, S. R., and Li, B.: In situ spectroscopy of the solar sorona, Astron. Astrophys., 482, 981-987, doi:10.1051/0004-6361:20079202, 2008.

Noci, G., Kohl, J. L., Antonucci, E., Tondello, G., Huber, M. C. E., Fineschi, S., Gardner, L. D., Naletto, G., Nicolosi, P., Raymond, J. C., Romoli, M., Spadaro, D., Siegmund, O. H. W., Benna, C., Ciaravella, A., Giordano, S., Michels, J., Modigliani, A., Panasyuk, A., Pernechele, C., Poletto, G., Smith, P. L., and Strachan, L.: First results from UVCS/SOHO, Adv. Space Res., 20, 2219-2230, doi:10.1016/S0273-1177(97)00895-8, 1997.

Ofman, L.: Source regions of the slow solar wind in coronal streamers, Geophys. Res. Lett., 27, 2885-2888, doi:10.1029/2000GL000097, 2000.
Ofman, L.: The origin of the slow solar wind in coronal streamers, Adv. Space Res., 33, 681-688, doi:10.1016/S02731177(03)00235-7, 2004.

Ofman, L. and Kramar, M.: Modeling the Slow Solar Wind during the Solar Minimum, SOHO-23: Understanding a Peculiar Solar Minimum ASP Conference Series 428, edited by: Cranmer, S R., Hoeksema, J. T., and Kohl, J. L., San Francisco: Astronomical Society of the Pacific, 321-324, 2010.

Ofman, L., Abbo, L., and Giordano, S.: Multi-fluid model of a streamer at solar minimum and comparison with observations, Astrophys. J., 734, 30, doi:10.1088/0004-637X/734/1/30, 2011.

Ofman, L., Abbo, L., and Giordano, S.: Observations and models of slow solar wind with $\mathrm{Mg}^{9+}$ ions in quiescent streamers, Astrophys. J., 762, 18, doi:10.1088/0004-637X/762/1/18, 2013.

Parker, E. N.: Interplanetary dynamical processes, New York, Interscience Publishers, 1963.

Raymond, J. C., Kohl, J. L., Noci, G., Antonucci, E., Tondello, G., Huber, M. C. E., Gardner, L. D., Nicolosi, P., Fineschi, S., Romoli, M., Spadaro, D., Siegmund, O. H. W., Benna, C., Ciaravella, A., Cranmer, S., Giordano, S., Karovska, M., Martin, R., Michels, J., Modigliani, A., Naletto, G., Panasyuk, A., Pernechele, C., Poletto, G., Smith, P. L., Suleiman, R. M., and Strachan, L.: Composition of Coronal Streamers from the SOHO Ultraviolet Coronagraph Spectrometer, Sol. Phys., 175, 645-665, doi:10.1023/A:1004948423169, 1997.

Riley, P., Linker, J. A., Mikic, Z., Lionello, R., Ledvina, S. A., and Luhmann, J. G.: A Comparison between Global Solar Magnetohydrodynamic and Potential Field Source Surface Model Results, Astrophys. J., 653, 1510, doi:10.1086/508565, 2006.

Sheeley, N. R., Wang, Y.-M., Hawley, S. H., Brueckner, G. E., Dere, K. P., Howard, R. A., Koomen, M. J., Korendyke, C. M., Michels, D. J., Paswaters, S. E., Socker, D. G., St. Cyr, O. C., Wang, D., Lamy, P. L., Llebaria, A., Schwenn, R., Simnett, G. M., Plunkett, S., and Biesecker, D. A.: Measurements of Flow Speeds in the Corona Between 2 and $30 R_{\otimes}$, Astrophys. J., 484, 472, doi:10.1086/304338, 1997.

Steinolfson, R. S. and Nakagawa, Y.: Dynamical Response of the Solar Corona. II. Numerical Simulations Near the Sun, Astrophys. J., 207, 300-307, doi:10.1086/154494, 1976.

Strachan, L., Suleiman, R., Panasyuk, A. V., Biesecker, D. A., and Kohl, J. L.: Empirical Densities, Kinetic Temperatures, and Outflow Velocities in the Equatorial Streamer Belt at Solar Minimum, Astrophys. J., 571, 1008, doi:10.1086/339984, 2002.

Zhao, X. P. and Hoeksema, J. T.: The Magnetic Field at the Inner Boundary of the Heliosphere Around Solar Minimum, Sol Phys., 266, 379-390, doi:10.1007/s11207-010-9618-0, 2010. 